\title{
Aspectos sociales de la introducción del color en la red de televisión colombiana ${ }^{1}$
}

\author{
Social aspects of the introduction of color in the \\ Colombian television network
}

\author{
Juan Arturo Camargo Uribe \\ Antonio García Rozo²
}

\section{Resumen}

En el artículo narramos aspectos del proceso de introducción del color en la red colombiana de televisión. Nuestro objetivo analítico consiste en problematizar la comprensión lineal característica de las narraciones históricas progresivas del cambio socio-técnico, mostrando una red heterogénea de circunstancias históricas, sociales, económicas, culturales, técnicas y políticas involucradas en el proceso, las cuales permiten explicar las alternativas tecnológicas consideradas y las trayectorias técnicas e institucionales resultantes. Las conclusiones de la revisión histórica sugieren que una comprensión sobresimplificada del cambio socio-técnico redunda en una baja capacidad para orientarlo de acuerdo con metas sociales ambiciosas.

\section{Palabras clave}

Educación en tecnología, ciencia, tecnología y sociedad, historia de Colombia, cambio tecnológico, innovación.

\section{Abstract}

In this paper, we report some aspects of the process of the introduction of color to the Colombian television network. Our analytical approach is to see lineal comprehensions, typical of progressive histories of technological change, as problematic. Consequently, we try to take into account heterogeneous arrays of the historical, social, economical, cultural, technical and political circumstances involved in the process. This approach allows us to explain the technological alternatives that were considered and the technical and institutional pathways that were effectively implemented. The historical review suggests that an oversimplified comprehension of socio-technical change gives rise to a low capacity to guide it with respect to more ambitious social goals.

\section{Key words}

Technology education, science, technology and society, Colombian history, technological change, innovation.

Artículo recibido el 14 de febrero de 2011 y aprobado el 19 de agosto de 2011

1 Este artículo se preparó en el marco del proyecto Alternativas socio-técnicas en la historia de la televisión en Colombia, financiado por la Vicerrectoría de Investigaciones de la Universidad de los Andes

2 Profesores del Grupo Tecnología y Sociedad de la Facultad de Ingeniería de la Universidad de los Andes.

Correos electrónicos: jucamarg@uniandes.edu.co, angarcia@uniandes.edu.co 


\section{Presentación}

A menudo escuchamos que el paso del "blanco y negro" al "color" cambió la historia de la televisión colombiana, dando a entender que se trató de una evolución lineal y positiva, sin tensiones sociales de ningún tipo. Sin embargo, la intención de este artículo es precisamente presentar una descripción más compleja de este proceso de cambio tecnológico, tratando de comprender las alternativas presentes, la relación de las dinámicas del cambio con los diversos intereses de los actores involucrados, las controversias que surgieron entre ellos, así como las grandes transformaciones institucionales que implicó el "color", las cuales dieron forma a la nueva red de TV.

Aparte de evidenciar las implicaciones del cambio tecnológico sobre la institucionalización de las telecomunicaciones, consideramos que este análisis puede beneficiar tanto a los educadores en tecnología como a tecnólogos, ingenieros y planificadores, aportando elementos para una comprensión enriquecida de la compleja red de circunstancias socio-políticas que afectan y son afectadas por el desarrollo de los sistemas tecnológicos.

A lo largo del artículo planteamos una narración histórica del cambio tecnológico en el sistema de televisión en Colombia, centrando la atención en el proceso de actualización, a finales de los setenta, del sistema monocromático al de color. Al hacerlo, discutimos algunas de las interpretaciones sobre impacto del cambio tecnológico en la historia de la televisión propuestas por analistas interesados en el tema en nuestro país, que se refieren al paso al color.

Entre las conclusiones destacamos, en primer lugar, el hecho sorprendente de que la selección de la norma estadounidense NTSC, hasta cierto punto, ha sido impuesta por la existencia de miles de televisores a color importados informalmente de los EE.UU. En segundo lugar, contradiciendo la opinión predominante de que se trató de un cambio repentino, mostramos que el paso de nuestra televisión al color involucró un proceso social y técnico prolongado, que comienza en los años setenta y finaliza hacia mediados de los ochenta. En tercer lugar, confirmamos que a pesar de ser un evento "puramente técnico" en apariencia, realmente aceleró el desplazamiento del control institucional del sistema, de manos del Gobierno a favor de empresarios particulares, lo cual vino acompañado de un incremento notable en la penetración del medio y la comercialización masiva del servicio público de televisión.

En el plano teórico-metodológico, el artículo ofrece algunos elementos a la discusión, derivados del análisis de Grandes Sistemas Tecnológicos (Hughes 1983, 1989, 1994; Winner 1993) y la Teoría Actor Red (Latour 1999; Callon 1989), que permiten analizar los vínculos sociales y tecnológicos. El texto también constituye una invitación a los lectores a emprender análisis similares de otros sistemas tecnológicos de nuestro país, que consideramos elementos claves en la comprensión del estilo tecnológico colombiano y un aporte a la construcción gradual de una identidad nacional bien definida en este aspecto.

\section{Introducción}

El año 1979 quedó registrado como el momento en que se introdujo la televisión a color en nuestro país, lo cual es considerado convencionalmente un progreso significativo -aunque tardío- en este sistema tecnológico, en el que Colombia se había adelantado originalmente a la mayoría de países de la región. Según la narración oficial, el cambio se produjo sin tropiezos tecnológicos y transformó drásticamente la industria, produciendo gran beneficio para la sociedad en general. En efecto, la introducción del color marcó una explosión de la industria en términos de cobertura 3 , capacidad y penetración. Las horas de programación se ampliaron poco después de la actualización y, en muchas regiones, la llegada del "color" coincidió con la introducción de un segundo canal.

Sin embargo, el proceso también trajo consigo profundas transformaciones en otros aspectos críticos del sistema socio-técnico de la televisión. Por ejemplo, la configuración institucional dejó de

3 Cobertura se refiere a las regiones geográficas donde llega la señal; capacidad, al número de canales; y penetración, al número de receptores disponibles. 
estar sometida a un control gubernamental cuasihegemónico para dar paso a un nuevo equilibrio de poder más afín a los intereses comerciales privados de programadores y anunciantes. Importantes instituciones públicas -como el Instituto Nacional de Radio y Televisión- perdieron importancia, y los programadores privados, respaldados por la experiencia adquirida con la producción de color desde 1974, se convirtieron en actores principales de la producción de televisión en el país.

Desde un punto de vista geopolítico, el color y especialmente el estándar NTSC adoptado, permitió a la televisión colombiana fortalecer sus vínculos con las redes de producción y distribución de contenidos de los Estados Unidos a través de satélite y por medio de la importación de video grabaciones. Este punto alteró el balance entre contenidos locales y extranjeros ( $60 \%$ y $40 \%$ respectivamente), invirtiéndolo a favor de los contenidos extranjeros en la misma proporción (Getino, 1998).

En este texto procuramos ofrecer un relato histórico matizado con elementos sociales y culturales, a través del cual hacemos referencia a la naturaleza conflictiva del cambio tecnológico, en contraposición a lo que es presentado tradicionalmente como un fenómeno lineal sin mayores controversias 4 . Este enfoque puede ser útil para los estudiantes y profesores de tecnología, al igual que para técnicos e ingenieros, quienes como constructores de sistemas tecnológicos deben reflexionar respecto al grado y tipo de control que tenemos sobre los grandes sistemas tecnológicos, sujetos a factores como el impulso tecnológico o la dependencia de la trayectoria, que reducen drásticamente el grado de libertad de los planificadores y diseñadores, para seleccionar la mejor tecnología.

También resulta interesante observar el impacto de factores estructurales (como la geografía, la concentración empresarial o la estructura institucional) en el ritmo del cambio y registrar la importancia de eventos contingentes (como la visita papal, el mundial de fútbol, el advenimiento de los satélites o el

4 El trabajo desarrolla algunas de las ideas formuladas en Williams (2003). desarrollo de la industria de televisión por cable). Argumentamos que el retraso en la actualización a color en Colombia fue consecuencia de los arreglos institucionales mixtos (entre entidades públicas y privadas) imperantes durante los primeros años. Contrariamente, la decisión final de cambio se relaciona más con una contingencia de mucho valor para la industria de los medios globales: la Copa Mundial de Fútbol de 1978 en Argentina; igualmente, con el compromiso establecido en los acuerdos de comercio de la Región Andina, en el que Colombia se comprometía a actualizar por completo su sistema de televisión antes de 1982.

Finalmente, un aspecto de carácter contingente que afectó notablemente la decisión final fue la existencia de miles de televisores en color y equipos de reproducción de video con estándar americano en los hogares colombianos. Estos habían ingresado tanto por el puerto libre de San Andrés como por las rutas del contrabando antes de 1979, a pesar de que ese año quedó fijado en la memoria oficial como el momento de la actualización.

En la siguiente sección examinaremos los planteamientos de algunos de los analistas colombianos que han abordado el tema de la actualización al color. Luego narraremos el proceso de cambio teniendo en cuenta las consideraciones socio-técnicas que emergen del análisis de fuentes primarias y secundarias 5 , y finalmente discutiremos los resultados del análisis en el contexto de las preguntas: ¿Cómo se dio el cambio en la tecnología en este caso en particular? ¿En qué medida los planificadores intervinieron en la configuración del cambio? Al concluir, nos referiremos a la posible utilidad de una mejor comprensión de los procesos de cambio social y tecnológico, incluyendo las implicaciones institucionales del mismo, refiriéndonos a la reciente selección de la norma europea para el sistema de televisión digital terrestre colombiano.

5 Las fuentes primarias del estudio incluyen memorias de los ministros de comunicaciones, informes anuales de los directores del Instituto Nacional de Radio y Televisión y revisión de la prensa, así como de artículos y estudios realizados durante los años setenta. También entrevistamos a algunas de las personas que estuvieron directamente involucrados con el cambio y discutimos con ellos las conclusiones preliminares. 


\section{Apropiación privada de asuntos de dominio público}

Antes de presentar nuestra visión del cambio tecnológico que estamos considerando, introduciremos su análisis refiriéndonos a las críticas más significativas que han formulado los analistas de los medios de comunicación en relación con la historia de la televisión colombiana. La bibliografía consultada incluye preguntas sobre la dinámica social de dicho sector y los motores del cambio técnico e institucional. Paradójicamente, a pesar de que muchos autores reconocen la importancia del cambio técnico, sus narraciones no suelen abordarlo de manera explícita, como sucede igualmente con el color.

La apropiación del dominio público por parte del sector privado es un tema común en el estudio de la historia de las instituciones colombianas. Según varios analistas ese proceso es concomitante con los cambios tecnológicos, y aunque no hay consenso en cuanto al momento y la magnitud del fenómeno, para la mayoría de ellos la televisión colombiana experimentó pronto dicha apropiación; específicamente se vivió de forma simultánea con el periodo político del Frente Nacional (1958-1974) y permitió a la industria colombiana de medios de comunicación y publicidad -a partir de mediados de la década de los sesenta (Fadul y Peñalosa, 1959)sacar la mejor partida de un sistema y una audiencia de televidentes, cuya costosa conformación inicial fue realizada con recursos públicos.

Para mayor claridad nos concentraremos en tres aspectos problemáticos. En primer lugar, el proceso de selección del estándar y el estándar elegido, que afectaron la magnitud y la distribución de las inversiones. En segundo lugar, el equilibrio entre la cantidad de programación de contenido nacional e internacional, que afectó los mercados de América Latina (por ejemplo las telenovelas argentinas fueron reemplazadas por las venezolanas [Fox, 1993]); y en el tercer lugar, destacamos el impacto del cambio tecnológico en el balance de poder entre las instituciones públicas y privadas que intervenían en la administración y explotación de los medios de comunicación, proceso que favoreció la expansión comercial privada (Vizcaino, 2004).

\section{La selección de la norma}

A pesar de que en octubre de 1978 la conformación de la comisión a cargo de la selección de la norma es presentada oficialmente como la marca fundacional de este "progreso", hasta donde tenemos conocimiento nadie ha propuesto una tesis acerca del desarrollo de los eventos. Herrán (1991) revisa el desarrollo inicial de las normas existentes (NTSC B/W en 1941; NTSC a color en1953; SECAM en 1959; PAL en 1963) y afirma que el desarrollo histórico de la televisión ha seguido a la tecnología. Sin embargo, al igual que otros analistas, no tiene en cuenta la trayectoria detallada y las implicaciones del proceso de selección del estándar. Hacemos hincapié en la peculiaridad de la naturaleza de la comisión y la percepción pública de su labor. Mientras que el público en general no era muy consciente de las implicaciones ${ }^{6}$, el gobierno tenía interés en favorecer la norma estadounidense NTSC; y la Embajada de Estados Unidos también. En contraste, muchos ingenieros consideraban que si el país había retrasado la entrada del color por casi una década, se debía aprovechar la oportunidad del ingreso tardío con el fin de elegir la opción más sólida para la geografía colombiana que, como era ampliamente reconocido, era el estándar alemán PAL. El gobierno resolvió la controversia con una cuidadosa conformación de la comisión, incluyendo miembros que tenían una visión amplia del cambio tecnológico. Ésta reconocía la importancia, tanto de las trayectorias recorridas como de otras consideraciones prácticas, sobre la geopolítica de las redes regionales de conocimientos técnicos y del comercio de contenidos.

\section{La producción de contenidos}

Las barreras asociadas con los estándares técnicos para el color afectaban el libre intercambio de material de vídeo en línea, imponiendo un formato de transferencia. A pesar de que los diferentes estándares no impiden del todo el intercambio de contenido comercial, la conversión era costosa y afectaba la calidad de la señal. La primacía de los

6 Entrevista con F. García (miembro del comité de selección), sostenida en Junio de 2009. 
contenidos norteamericanos era una característica bien arraigada en el sistema colombiano, el cual no se quería alterar, y por lo tanto la introducción del estándar alemán tenía un costo geopolítico elevado, que los norteamericanos no estaban dispuestos a soportar. La Embajada de Estados Unidos, en nombre de los industriales estadounidenses, organizó una demostración de lujo de la norma NTSC en la sede diplomática estadounidense en Bogotá7.

Inicialmente, el aspecto más sobresaliente del cambio de formato en lo relacionado con la producción de contenidos locales fue el desplazamiento de los programas nacionales, la mayoría de ellos producidos en instalaciones del gobierno, a favor de un mayor porcentaje (60\%) de contenidos extranjeros. Con el paso del tiempo, gradualmente se alcanzó una distribución de cincuenta-cincuenta por ciento entre contenidos extranjeros (sobre todo provenientes de Estados Unidos) y la producción colombiana (Teletexto, 1994)8.

La distribución del trabajo entre el Estado y los actores privados para la producción de contenidos a nivel local también experimentó un cambio drástico. Los programadores particulares aumentaron su participación, y cuando la obligación legal de producir sus programas en las instalaciones del gobierno fue retirada, llegó a ser superior al 70\%. Este aspecto también estuvo influenciado por el hecho de que, mientras que los estudios privados habían estado trabajando con equipos a color desde varios años antes del cambio, el Instituto Nacional de Radio y Televisión tuvo que recorrer una dolorosa curva de aprendizaje prácticamente desde ceros.

\section{El balance entre lo público y lo privado}

Según Elizabeth Fox (1993), el sistema colombiano es uno de los casos más exitosos de monopolización de la televisión en América Latina. Fox considera la mayoría de ellos como "productos de una compleja interacción de gobiernos débiles, el autoritarismo y el populismo, junto con las condiciones del mercado

7 Entrevista con ingeniero F. Garcia (miembro del comité de selección), sostenida en Junio de 2009.

8 Entrevista con el ingeniero R. Mejia (Abril 2009) nacional e internacional, y la exclusión de importantes fuerzas sociales». Para Herrán, el estatus de "servicio público" de la industria de la televisión es una ficción que esconde su carácter exclusivamente comercial. Vizcaíno, por su parte, argumenta que el sistema se compone de dos industrias independientes producidas por la división del trabajo; una red pública, educativa y orientada políticamente, y una empresa privada dedicada a los negocios rentables de la información y el entretenimiento. Estas tesis no son nuevas; Bibliowitz (1979) ya había señalado, en un estudio de los años setenta, que en la televisión colombiana los bienes públicos habían sido tomados por los intereses privados.

Según Herrán, el control económico sobre la televisión da cuenta del poder monopolístico ejercido por los propietarios de la industria de los medios sobre la televisión colombiana. También afirma que la tecnología jalona los cambios en la televisión más rápido que en cualquier otro medio, y que es difícil para los gobiernos mantener la regulación actualizada. Sin embargo, Herrán no tiene en cuenta la influencia de los medios de comunicación internacionales y concuerda con los que explican la historia del sistema colombiano de televisión como el resultado directo de la estructura económica capitalista y las luchas entre gobierno, empresarios y políticos.

Vizcaíno, por su parte, articula el desplazamiento en favor del interés privado con la transición tecnológica impulsada a modo post-fordista de producción en la industria de la televisión (Vizcaíno, 2004). En consecuencia, relaciona el giro comercial de mediados de los ochenta, con la diversificación de la red causada por los servicios de cable, el despliegue masivo de antenas parabólicas para recepción satelital y la aparición de los canales regionales no comerciales en Antioquia y en la Costa Atlántica. Consecuentemente, Vizcaíno denuncia una falsa dicotomía entre las explicaciones basadas en la tecnología y las basadas en la privatización, pues para él estos constituyen en realidad fenómenos complementarios. Con relación al mismo punto, E. Fox y Anzola (1988) han sugerido que el surgimiento 
de la configuración monopolística en la industria de los medios de comunicación en Colombia sólo tuvo lugar después del proceso de liberalización iniciado en los años noventa; mientras que Vizcaíno resalta la importancia de la estación satelital terrena, inaugurada en 1971, para la importación de contenidos de televisión, como el campeonato mundial de ciclismo en Italia, ganado por un colombiano en 1971, o la extensión de la señal Nacional de Colombia a San Andrés (1974) y Leticia (1976) (Téllez, 1979).

\section{Relato histórico}

El siguiente relato es fruto de un proyecto en el que se estudiaron seis momentos socio-técnicos destacados de la historia de la televisión nacional, definidos por los autores. Inaugurado en 1954, el sistema de TV colombiano mantuvo una forma institucional muy centralizada por más de treinta años. En ella se mezclaron los intereses contradictorios de una agencia gubernamental encargada de la programación y de la transmisión del sistema, que concebía la televisión como servicio público; y los intereses de los programadores privados y la industria del mercadeo, que buscaba beneficios económicos. Esto se reflejó en una convivencia estable entre los intereses públicos y políticos, y los intereses comerciales privados.

Después de un período inicial de control directo de la televisión desde la Presidencia de la República durante el período bipartidista del Frente Nacional (1958-1974), el control político de las noticias y otros programas de información fueron compartidos por los dos partidos dominantes, mientras que los intereses comerciales tomaron el control de las franjas de entretenimiento y las oportunidades de negocio con la publicidad. El Instituto Nacional de Radio y Televisión operaba la Cadena Nacional o Canal Uno, de muy amplia cobertura a nivel nacional, junto a otros dos canales, uno con tinte comercial y un canal educativo, ambos con una cobertura limitada a la región andina central del país. Todos ellos utilizaban el formato americano NTSC para televisión en blanco y negro, que para entonces era el único formato de televisión que operaba con los servicios eléctricos a 60 hertzios existentes en Colombia. Además, la red de televisión colombiana había logrado una cobertura nacional desde sus comienzos, gracias a las altas cordilleras y el uso innovador de transmisión con repetidores, operando en las mismas frecuencias utilizadas para distribuir la señal a los receptores.

Durante los años setenta el gobierno, reacio a perder el control del servicio, ejerció una política muy cerrada de orientación de la televisión. Esta, operada de manera formal en un esquema mixto, distribuía las responsabilidades entre el Instituto Nacional de Radio y Televisión, oficina pública encargada "de un servicio considerado público» y la industria de los medios, que explotaba la televisión como un elemento de entretenimiento y mercadeo. El estado poseía la infraestructura y el conocimiento de la totalidad de los componentes del sistema de televisión y alquilaba tanto los servicios de producción, como los "espacios" en las franjas de transmisión de la cadena nacional. En esos espacios se programaban espectáculos de entretenimiento y se revendía el tiempo a la industria del mercadeo para transmitir publicidad. Los programas de noticias eran producidos en los estudios del gobierno y se distribuían entre empresarios liberales y conservadores.

Los componentes de transmisión y distribución conformaban una extensa red que cubría las áreas más pobladas de un inmenso territorio, la cual habría sido más dispersa y fragmentada sin esta cadena de televisión, puesta en marcha por el gobierno fuerte del ingeniero y general Gustavo Rojas Pinilla en los años cincuenta. Ese sistema fue una gran innovación en su momento, pues hacía uso de la cordillera de los Andes para retransmitir la señal en sólo siete largos saltos, hecho que permitió cubrir más de 900 kilómetros desde Bogotá hasta Barranquilla, con unas pocas estaciones repetidoras (Quijano, 1951).

La red también creció hacia el sur, y como resultado de esto se logró un sistema que cubría las ciudades principales y secundarias, más del $80 \%$ de las zonas pobladas (sobre todo la región andina y la costa del Caribe). A pesar de ello, la huella de esta cadena de televisión dejaba por fuera vastos territo- 
rios como los Llanos Orientales, la costa del Pacífico y la Amazonía. A pesar de esta amplia cobertura, la penetración real del servicio era baja. Menos del $30 \%$ de los hogares tenían televisor, y la necesidad de energía eléctrica imponía severas limitaciones a la penetración del servicio.

A principios de los años setenta todo esto empezó a cambiar. Los gobiernos bipartidistas habían finalizado en 1974 y la industria de la producción local comenzó a percibir los beneficios de una industria de la televisión más flexible y dinámica (Nueva Frontera 1978). Las redes internacionales y nacionales empezaron a establecer vínculos en ambas direcciones. Un ejemplo significativo fue la visita papal de 1968 que se distribuyó a las redes extranjeras (mercados) a través del servicio de televisión del Vaticano, mediante una estación satelital terrena traída especialmente por el pontífice para ese evento.

Un año más tarde, el programador privado (y constructor pionero del sistema de televisión) Fernando Gómez A., se comprometió a transmitir en vivo y en directo para el público colombiano las imágenes del alunizaje del Apollo11, para lo cual la señal tenía que ser retransmitida de las emisiones de la NASA en los Estados Unidos. Tras considerar el uso de un repetidor en un avión, finalmente el enlace se logró, superando grandes dificultades, por medio de repetidores terrestres (de nuevo gracias a la cordillera de los Andes) encadenados a la estación terrena que operaba la televisión venezolana en Caracas. Aunque estos episodios fueron generados y transmitidos en color para las redes extranjeras, cuando eran retransmitidos para el sistema colombiano la señal se convertía (degradada) a blanco y negro. Unos años más tarde, este cambio se hizo más evidente, pues la nueva estación satelital terrena de Chocontá permitió la recepción continua de señales de televisión extranjera en color. En los años setenta, el gobierno introdujo una tercera cadena de televisión con fines educativos, respaldada con recursos financieros de la Alianza para el progreso.

Además de que el sistema había crecido lentamente por más de veinte años, el conjunto de producción, programación, emisión y las actividades de transmisión fueron controladas exclusivamente por el Instituto Nacional de Radio y Televisión, en cuyos estudios se habían desarrollado para la televisión en blanco y negro exclusivamente. No obstante, a partir de 1971, la señal de televisión en color llegó al territorio colombiano a través de la estación satelital terrena instalada por el gobierno cerca de Bogotá. Las instalaciones de la estación de Chocontá permitieron intercambiar señales de televisión a color de agencias de noticias internacionales y esto permitió el establecimiento de acuerdos de intercambio de noticias que exigían el uso de formatos de color. Es probable que ello alentase al gobierno a dar permiso para construir sus propios estudios, en 1974, a las tres empresas líderes en producción . Estas empresas, asociadas para crear las primeras instalaciones privadas de producción de televisión en el país, a pesar de la limitación de la red existente para la distribución monocromática en blanco y negro, decidieron adaptar las instalaciones para la producción de televisión a color, debido a su interés por llegar a mercados internacionales y por presionar el cambio en la red colombiana (Vizcaíno, 1975) ${ }^{9}$.

El periodo del Frente Nacional concluyó en 1974, pero la inercia bipartidista en el estilo de gobierno explica la persistencia prolongada de un esquema de televisión centralizada y monocromática hasta finales de los años ochenta. A pesar de que las narraciones convencionales sobre la modernización de las redes y las facilidades de producción para acoger la televisión a color describen usualmente un proceso lineal, nuestra investigación muestra que durante los siete años previos al cambio se debatieron acaloradamente otras inversiones alternativas, y que ésto, como usualmente ocurre con los cambios tecnológicos, no estuvo libre de controversias y realmente implicó intensas luchas por parte de un conjunto de empresarios decididos a vencer la resistencia al cambio de la iglesia católica y los sindicatos de trabajadores de las telecomunicaciones, quienes se oponían al cambio.

9 Vizcaíno menciona que Gravy, productora privada, realizó la popular telenovela La Vorágine en color en 1975. 
Nada de esto es sorprendente, dado que la actualización requería una inversión superior a los cien millones de dólares, y tanto los costos como los beneficios estaban distribuidos inequitativamente entre los involucrados. La inversión por parte de los ciudadanos, que correspondía al cambio de los receptores, representaba cerca del ochenta por ciento del total, mientras que la inversión en cabeza del gobierno, correspondiente a la actualización de las redes de transmisión y distribución, y la modernización de las facilidades de producción del Instituto Nacional de Radio y Televisión, cubrían el veinte por ciento adicional. Como se mencionó antes, los programadores particulares, quienes eran los principales beneficiarios, habían equipado sus estudios con la tecnología de color desde su construcción en 1974.

Durante la primera presidencia no bipartidista del liberal Alfonso López M., el gobierno propuso un acto legislativo para permitir la creación de emisoras privadas locales que operarían en color, pero en ese proyecto no se permitía la interconexión entre ellas. Aunque la iniciativa fue derrotada en el Congreso, López introdujo a partir de 1976 una nueva práctica en la administración de TV, reservando un espacio de noticias para uso gubernamental; hecho que ha sido considerado factor central en la elección para presidente del liberal Julio Cesar Turbay un año más tarde. Esta decisión fue duramente criticada, pues rompió el pacto tácito del gobierno de no intervenir de manera directa en la explotación comercial del servicio, ni de caer en una polarización política del control de los medios de comunicación.

Además del alto costo del cambio, encontramos evidencia de que en este participaron otros elementos importantes como la percepción negativa que algunos analistas plantearon sobre el tema, porque consideraban que era una innovación costosa, pobremente justificada. Para ellos existían prioridades de inversión más acuciantes, como los proyectos de abastecimiento de agua, de salud o de educación. $\mathrm{Al}$ respecto se plantearon otras consideraciones macro-económicas, como el impacto negativo que podría tener el cambio en la balanza de pagos del país, teniendo en cuenta que el gran número de receptores de televisión nuevos que se necesitarían, tenían que ser importados o comprados por partes para ensamblar en el extranjero. Por otro lado, los comentaristas más críticos consideraron que la actualización también tendría inconvenientes en términos sociales, porque acentuaría la propagación de una comunidad dividida entre quienes eran capaces de invertir en un costoso aparato de TV y los que tenían que permanecer con la vieja tecnología en blanco y negro.

En agosto de 1978 la posesión presidencial de Julio C. Turbay fue transmitida en color -con estándar NTSC- para Bogotá y Cali, y semanas más tarde se creó el Comité Asesor para el estudio de los sistemas de televisión a color, encargado de presentar al Gobierno Nacional, en un plazo de 45 días, sus recomendaciones sobre la selección del sistema que debería adoptar el Instituto Nacional de Radio y Televisión. El comité estaba integrado por Germán Vargas, director del Instituto, tres senadores, tres diputados, el decano de la Facultad de Ingeniería Electrónica de la Pontificia Universidad Javeriana, el decano de la Facultad Ingeniería Electrónica de la Universidad Distrital de Bogotá, dos ingenieros y el prestigioso médico e inventor colombiano Jorge Reynolds.

El comité de selección del estándar se puso a trabajar con urgencia para tomar una decisión apresurada por los compromisos que el gobierno había asumido dentro de los acuerdos regionales del Pacto Andino y la necesidad de sustituir la red de transporte existente, que ya había cumplido su ciclo de vida (20 años). Pese a la recomendación final de la comisión asesora del gobierno, que favorecía el estándar NTSC, la opinión pública conoció los desacuerdos sobre la calidad técnica del sistema seleccionado y se rumoraba que la embajada norteamericana había participado activamente en el proceso. La selección estándar fue seguida por un despliegue rápido y por la explosión de la industria de la televisión en términos de producción, número de horas de programación e inversiones en publicidad, las cuales crecieron hasta cifras nunca antes vistas. Paradójicamente, el "padre" de la televisión colombiana y propietario de la programadora líder, 
RTI, se pronunció a favor de sistema de color PAL, a pesar del hecho de que los estudios de RTI (de su propiedad) estaban equipados para la producción en estándar NTSC. No menos interesante es el hecho de que Joaquín Quijano C., quien era el ingeniero líder de la instalación de la red original y experto en telecomunicaciones de gran reputación, también criticó la no adopción del estándar PAL, que él consideraba la opción obvia desde una perspectiva "técnica".

Como era de esperarse, el color llegó a las grandes ciudades con rapidez, y su expansión fue acompañada por la mejora de la cobertura del segundo canal a nuevas regiones, como la costa Atlántica, Quindío y Nariño, entre otras.

\section{Discusión socio-técnica}

La tecnología es un proceso complejo, con un origen claramente relacionado con causas anteriores y consecuencias futuras, y no un hecho aislado, ni el resultado de una discusión racional y planeada entre diversos actores sociales. Es, simultáneamente, un proceso orientado y dependiente de la trayectoria, que podría describirse como un fenómeno social similar, en cierta medida, al lenguaje, por no depender de ningún control individual directo, sino ser el producto de los cambios graduales derivados del uso cotidiano. Hemos visto cómo la perspectiva histórica nos permite percibir cambios significativos en la percepción pública de los temas que estaban en juego durante este proceso de cambio tecnológico. Algunos de ellos fueron reconocidos abiertamente durante los procesos de decisión y selección del estándar, mientras que otros, no menos importantes desde el punto de vista socio-técnico, fueron ignorados o pasaron inadvertidos en su momento.

El cambio en este relato es un ejemplo de impulso de las tecnologías, en un sistema tecnológico altamente inercial. Esto se puede atribuir, en última instancia, a la madurez de las redes internacionales de comercio (tanto en la comercialización como en las industrias de contenidos), a la nueva disposición de poder entre los partidos políticos y de gobierno -que terminó con la adquisición por parte del Estado de su propio noticiero para competir con los demás- y también a los cambios en las prioridades del gobierno. La selección del estándar, por otra parte, pone de manifiesto el peso de miles de equipos de Betamax-TV en color, buena parte de ellos importados de contrabando, y el despertar de una gran industria de importación -ilegal- de contenidos.

Es probable que el aspecto más relevante para la selección de la norma NTSC haya sido la inmediatez de los enlaces de televisión vía satélite con la red norteamericana, aspecto que también tuvo un lado informal (comercio de señales parabólicas incidentes) en los años ochenta, y que propició un proceso muy grande de diversificación de la red. Sin embargo, la posterior invasión de telenovelas del Brasil demostró que las diferencias en estándar no representaban un obstáculo para el comercio de contenidos, y es tentador afirmar que la penetración posterior de antenas parabólicas satelitales y contenidos estadounidenses a través de las redes de cable, pudo haberse disminuido drásticamente con la adopción del estándar de color europeo.

También hemos visto que el color fue un momento de cambio crítico, que marcó la consolidación de la privatización y la explosión de los medios de comunicación. El proceso tuvo un lado importante, informal, no planificado (Rey es el único que lo ha señalado antes de nosotros), así como un estilo local que sólo puede verse por medio de un relato histórico muy resumido, como el que hemos presentado en este artículo.

Considerando la pregunta sobre el efecto de privatización con el cambio al color, podemos decir que el proceso favoreció a los programadores comerciales, ya que fueron capaces de ampliar el horario de programación, la cantidad de contenido importado y el volumen de horas de televisión producidos en sus propios estudios. En consecuencia, se las arreglaron para reducir la costosa dependencia de los estudios de producción públicos.

Aunque se requiere investigación adicional para describir con precisión este efecto, tenemos suficiente evidencia para afirmar que también hubo un impacto drástico y positivo en las cifras económicas de las empresas, y que esto favoreció más a las asociaciones privadas que a las del sector público. Ello se debió al incremento de las tarifas de publicidad, 
correspondiente al incremento de los índices de audiencia (derivado del aumento drástico de los aparatos de televisión y la atracción que representaba la novedad del color), mientras que el número de horas de transmisión en el sistema público se redujo y las tarifas cobradas por el Instituto Nacional de Radio y Televisión no reflejaban las distorsiones en el comportamiento de la audiencia producidas por el color. El efecto diferencial en la producción de contenidos, discutido antes, también afectó el equilibrio entre el subsistema privado y el público.

Las industrias de la publicidad y el mercadeo fueron las beneficiarias directas de la actualización, puesto que el cambio les brindaba oportunidades mucho más amplias para la comercialización de productos, no solamente con más eficacia, sino también, y más importante, porque el número de televisores, el tiempo de programación y el volumen de negocios se multiplicaron geométricamente durante los siguientes años.

Desde el punto de vista de los programadores, también hubo diferencias importantes entre aquellos que tenían acceso a las instalaciones privadas (los tres principales) en cuyo caso el resultado fue claramente positivo, y los que permanecían utilizando las instalaciones públicas para la producción, quienes tuvieron que recorrer una larga curva de aprendizaje antes de alcanzar la capacidad para competir con éxito en la realización de productos para la televisión a color. Si bien los estudios públicos del Instituto Nacional de Radio y Televisión se mejoraron gradualmente, nunca obtuvieron el mismo nivel competitivo obtenido por las instalaciones privadas de producción.

\section{Consideración final}

Nuestra capacidad para diseñar y orientar los procesos de cambio técnico parece ser hoy tan baja para asegurar la gobernabilidad y la participación social plural, como lo fue en los años setenta. Aunque la complejidad tecnológica y la conspiración de fuerzas capitalistas globalizantes contribuyen a esta falta de control, nosotros la atribuimos también a una pobre comprensión de la dinámica socio-técnica. Esto dificulta la introducción de un conjunto más amplio de objetivos sociales en el diseño de los sistemas tecnológicos, dejando libre el campo para el surgimiento de un control comercial agresivo de la red. Con este trabajo esperamos contribuir a la construcción de un conocimiento local que permita a las fuerzas civiles, incluyendo profesores, tecnólogos, ingenieros y otros profesionales, participar en las discusiones tecnológicas que influyen profundamente en la configuración de nuestra sociedad. Una vez que comprendamos a fondo los procesos sociales de cambio técnico, podremos empezar a construir sistemas tecnológicos más exitosos en términos económicos, sociales y culturales. 


\section{Bibliografía}

Bibliowicz, A. (1979). Lo público es privado: un análisis de la televisión colombiana. New York: Ed. Conell. Ithaca.

Callon, M. (1989). The sociology of an actor-network: the case of the electric vehicle. En: Bijker, W., Hughes, T. and Pinch, T. (Eds.). The social construction of technological systems: new directions in the sociology and history of technology. Cambridge: The MIT Press.

Fadul, M. y Peñalosa, E. (1959). Estudio económico sobre la Radiotelevisora Nacional. Bogotá.

Fox, E. y Anzola, P. (1988). Politics of regional television in Colombia. En: Media and politics in Latin America. Ed. Fox, E. London: Sage publications.

(1993). International relations and national policies of Latin American broadcasting. Tesis Doctoral. En: International relations. The American University, Faculty of the School of International Service. Washington.

Getino, O. (1998). Cine y Televisión en América Latina. Producción y mercados. Buenos Aires: Ediciones Ciccus.

Hughes, T. (1983). The style of evolving systems. En: Networks of power. Electrification in western society. Cambridge MA: Johns Hopkins Press.

(1989). The evolution of large technological systems. En Bijker, W., Hughes, T. and Pinch, T. (Eds.) Social construction of technological systems: new directions in the sociology and history of technology. Cambridge MA: The MIT Press.
(1994). Technological Momentum. En: Marx, Leo and Smith, Roe (Eds.), Does technology drive history? The dilemma of technological determinism. Cambridge, MA: The MIT Press.

Latour, B. (1999). On recalling ANT. En: Law, J. and Hassard, J. (Eds.). Actor network theory and after. Wiley-Blackwell.

Quijano, J. (1951). Una red nacional de telecomunicaciones. Resumen de un proyecto. En: Anales de Ingeniería. No. 632. Vol.58. Año: Oct. Dic. 1951.

Revista Nueva Frontera (1978). TV color de hormiga. Sept.-Dic. 1978. Bogotá.

Revista Teletexto (1994). Inravisión. El futuro de Inravisión.

Téllez, H. (1979). Veinticinco años de televisión colombiana. RTI. Bogotá.

Vizcaíno, M. (2004). La legislación de televisión en Colombia: entre el Estado y el mercado. Historia Crítica. Rev. No. 28. Uniandes, Bogotá.

Williams, R. (2003). Television: technology and cultural form. London-New York: Routledge.

Winner, L. (1993). Social constructivism. Opening the black box and finding it empty. En: Science as Culture, 3(16). 
